\title{
Mão traumatizada - Atualização no primeiro atendimento
}

\section{Traumatized Hand - Update at the First Visit}

\author{
Flaviana Busignani da Silva1이 Giana Silveira Giostri2 ${ }^{20}$ \\ ${ }^{1}$ Membro do Grupo de Cirurgia da Mão, Hospital Universitário Cajuru, \\ Curitiba, Paraná, Brasil \\ 2 Professora Adjunta da Escola de Medicina, Pontifícia Universidade \\ Católica do Paraná, Curitiba, Paraná, Brasil \\ Endereço para correspondência Flaviana Busignani da Silva, Grupo de \\ Cirurgia da Mão, Hospital Universitário Cajuru, Avenida São José, 300, \\ Cristo Rei, Curitiba, Paraná, 80050-350, Brasil \\ (e-mail: flaviana.busignani@gmail.com).
}

Rev Bras Ortop 2021;56(5):543-549.

\section{Resumo}

Palavras-chave

- traumatismos da mão

- emergências

- fraturas ósseas
A mão é o segmento mais exposto ao trauma, com grande volume de atendimento em serviços de urgência e emergência. Portanto, faz-se necessário que os médicos plantonistas tenham conhecimento primordial para a conduta eficaz frente a essas lesões. Neste artigo, revisaremos as principais afecções e orientações de condutas.

The hand is the segment most exposed to trauma, with a large volume of care in urgent and emergency services. Therefore, it is necessary that physicians on duty have the essential knowledge to effectively manage these injuries. In the present article, we will review the main conditions and conduct guidelines.

\section{Introdução}

A mão é o segmento mais exposto ao trauma, e corresponde a parcela expressiva da procura nos serviços de emergência: cerca de $20 \%$ do número de atendimentos em geral. ${ }^{1-4}$ Esse número de lesões se deve à constante exposição aos ambientes de trabalho, doméstico, ou de lazer. Nesse sentido, há predominância de traumas na mão em todas as faixas etárias e grupos populacionais. ${ }^{5-11}$

No trabalhador economicamente ativo, os traumas na mão, que envolvem um período de tratamento e reabilitação, implicam perdas temporárias ou permanentes, e resultam em custo social e econômico. ${ }^{12}$ A conduta adequada, sobretudo na primeira abordagem, otimiza o processo de recuperação funcional da mão e evita sequelas indesejáveis.
Este artigo tem por objetivo revisar os principais traumas emergenciais na mão e orientar sobre o primeiro atendimento, com ênfase em pontos fundamentais que possam auxiliar os profissionais envolvidos no atendimento.

\section{Avaliação primária}

A anamnese adequada, com destaque para o mecanismo do trauma, é primordial para o diagnóstico e a conduta corretos. Nesse processo, são avaliados os fatores causais e o ambiente da ocorrência, que são úteis para o delineamento da extensão das lesões visando o tratamento necessário. ${ }^{13}$

Atenção peculiar deve ser dada ao paciente politraumatizado, em que o trauma de extremidades pode ser menos evidente do que as lesões sistêmicas graves, principalmente recebido

07 de Março de 2020

aceito

15 de Junho de 2021
DOI https://doi.org/

10.1055/s-0041-1735173. ISSN 0102-3616. (c) 2021. Sociedade Brasileira de Ortopedia e Traumatologia. All rights reserved.

This is an open access article published by Thieme under the terms of the Creative Commons Attribution-NonDerivative-NonCommercial-License, permitting copying and reproduction so long as the original work is given appropriate credit. Contents may not be used for commercial purposes, or adapted, remixed, transformed or built upon. (https://creativecommons.org/ licenses/by-nc-nd/4.0/)

Thieme Revinter Publicações Ltda., Rua do Matoso 170, Rio de Janeiro, RJ, CEP 20270-135, Brazil 
se o paciente estiver incapaz de sinalizar queixas. ${ }^{14} 0$ exame físico deve ser repetido com maior detalhamento após avaliação inicial e estabilização do quadro geral, seguindo os princípios do programa Suporte Avançado de Vida no Trauma (Advanced Trauma Life Support, ATLS, em inglês).

\section{Exame físico geral das mãos}

O exame físico geral das mãos deve ser feito da seguinte forma:

- Inspeção geral: ferimentos, edema, equimoses, postura do membro, deformidades angulares (dedos fletidos devem apontar para o tubérculo do escafoide);

- Teste de mobilidade passiva e ativa (amplitude de movimento). Avaliação isolada de cada dedo. Pesquisar integridade tendínea; e

- Testes neurológicos motor e sensitivo, de forma comparativa com o lado não lesionado.

\section{Exames complementares}

$\mathrm{Na}$ emergência, a radiografia é o exame complementar mais indicado, no qual se identificamas principais lesões quando realizado em projeções adequadas ao local de suspeita clínica. Existem particularidades em relação às imagens radiográficas da mão devido à sobreposição e ao número de ossos. Deve-se atentar para as projeções na forma correta a serem solicitadas conforme especificado a seguir:

- DEDOS: posteroanterior (PA) e de perfil, centradas no dedo;

- MÃO: PA, oblíqua e de perfil (nesta, são mais bem visualizadas as angulações em plano coronal e fraturas da base);

- OSSOS DO CARPO: PA e de perfil do punho; e

- Casos especiais:

- na suspeita de fratura de escafoide - PA com desvio ulnar da mão; e

- na lesão ligamentar escafossemilunar - PA com as mãos cerradas (pencil grip). ${ }^{15,16}$

É importante lembrar que as projeções radiográficas contralaterais podem auxiliar na comparação das estruturas e no diagnóstico das lesões, e que, após a abordagem inicial, outros exames de imagem podem ser necessários para firmar o diagnóstico de lesões não demonstradas na radiografia, ou até mesmo para graduar as já identificadas.

\section{Conduta nas lesões específicas}

\section{Pele}

A pele dorsal da mão é fina, pouco queratinizada, e proporciona grande deslizamento, mas pouca resistência à absorção de impactos. Já a porção palmar apresenta pele espessa e com grossa camada de queratina. Essa pele é bastante resistente à pressão; entretanto, a aderência na fáscia palmar dificulta seu deslizamento. ${ }^{17}$ Essas características demandam alguns cuidados frente a ferimentos e suturas na mão: ${ }^{17}$

- Feridas no dorso da mão podem cursar com lesão de tendões extensores: testar extensão completa das metacarpofalangeanas (MFs);
Suturas não devem ocasionar tensão: principalmente na pele palmar, em que há pouca resistência à tração;

- Atenção às linhas de força relacionadas aos movimentos de flexoextensão de dedos e mão: feridas longitudinais às pregas de flexão na palma terão mais tendência à retração cicatricial com limitação da extensão completa dos dedos;

- Feridas nos dedos: garrote cuidadoso na base do dedo auxilia no controle do sangramento e detalhamento da lesão;

- Pontos de sutura em feridas de mão e dedos devem ser retirados após 15 dias;

- Curativo com gaze seca deve permanecer enquanto os pontos da sutura forem mantidos;

- Após a retirada dos pontos de sutura: orientar cuidados com cicatrização (hidratação e mobilização);

- Cicatrização por segunda intenção: áreas pequenas, sem possibilidade de aproximação completa das margens, e sem exposição de osso, nervo, vaso, e tendão sem bainha;

- Retalhos locais: o retalho popularizado por Atasoy (em VY) é indicado preferencialmente para perdas digitais e amputações transversas da extremidade;

- Perdas extensas de pele ou com exposição de estruturas específicas: realizar primeiros cuidados de limpeza e curativo, orientando o paciente para os eventuais procedimentos seguintes (enxertos e retalhos).

\section{Unha}

Traumas compressivos nas unhas são muito comuns. Em crianças de baixa idade, de 0 a 6 anos, é o local de maior trauma na mão. ${ }^{18,19}$ Avulsões da unha devem ser reinseridas, com utilização de ponto em "U" orientando a extremidade do dedo. $^{20}$

Recomenda-se a drenagem asséptica de hematomas subungueais que ocupem mais de $25 \%$ do leito, embora alguns autores citem 50\%. ${ }^{21-23}$ Hematomas maiores do que 50\% associados à fratura sugerem lesão de leito, e considera-se a retirada da unha para reparo com fios de sutura finos (de 6.0 ou 7.0), que proporciona crescimento adequado e aderência da unha. $\mathrm{O}$ uso de magnificação é aconselhado devido à delicadeza das estruturas.

\section{Tendões}

Até mesmo pequenos ferimentos na mão causam lesões tendíneas, com índices elevados de negligência diagnóstica. Portanto, o exame físico deve ser individualizado para cada grupo muscular: flexores superficiais e profundos dos dedos, extensores das MFs e das interfalangeanas (IFs), músculos tênares (mobilidade do polegar), hipotênares (mobilidade do quinto dedo), lumbricais (flexão da MF) e interósseos (adução e abdução). Ressalta-se a frequente associação da lesão de nervos digitais em lesões de tendões flexores.

Na identificação da lesão tendínea, segue-se a programação para a tenorrafia, a ser realizada precocemente. Determinadas lesões, como no dorso da mão, são facilmente acessadas durante $o$ atendimento emergencial, e permitem sutura sem grande dificuldade técnica, desde que obedecendo o uso do fio de sutura inabsorvível com agulha atraumática. Lesões em outros locais, como nos tendões 
flexores nas zonas I e II de Verdan (túnel osteofibroso), demandam experiência técnica e aplicação de protocolo específico para reabilitação funcional da mão. Nesses casos, é importante a condução inicial com limpeza da ferida, sutura da pele, curativo, e orientação ao paciente sobre a necessidade da tenorrafia (-Figura 1).

A lesão tendínea mais comum é a deformidade conhecida como "dedo em martelo", provocada de forma costumeira por trauma axial. ${ }^{4}$ A radiografia de perfil do dedo acometido é mandatória para a pesquisa de fratura associada. 0 tratamento do "martelo" fechado, desde que ausente a subluxação palmar da falange distal, apresenta bom resultado com o uso contínuo da tala de alumínio ou órtese pré-fabricada, mantendo leve hiperextensão da articulação distal do dedo (-Figura 2). As trocas precisam ser supervisionadas, e a imobilização, mantida por seis a oito semanas. Quanto maior for a flexão inicial da IF distal, maior será a lesão da banda extensora terminal e suas expansões. Nesses casos, e principalmente se houver subluxação da falange distal, recomenda-se redução e fixação transarticular com fio de Kirschner de $1,0 \mathrm{~mm}$. Os mesmos parâmetros aplicam-se na lesão da banda central fechada - "dedo em botoeira", que ocorre na IF proximal do dedo. A imobilização deve ser realizada com tala apenas na articulação IF proximal, mantendo-a em extensão. 24,25
Tais lesões fechadas do aparelho extensor dos dedos, quando diagnosticadas em até duas semanas do trauma, são passíveis de tratamento conservador com bom resultado. ${ }^{24}$

\section{Nervos}

Semelhante à lesão de tendões, as lesões neurológicas podem passar despercebidas. Ferimentos pequenos ou puntiformes, alteração de sensibilidade falsamente inferida apenas ao edema, ou ausência de teste antes de anestesia da sutura são razões para a negligência dessas lesões.

O exame minucioso e comparativo, testando movimentos e sensibilidade (o paciente de olhos fechados percebe melhor as diferenças), auxilia na identificação de uma lesão nervosa. Se houver, esta deve ser reparada precocemente com técnicas de microcirurgia, de preferência em prazo inferior a três semanas do trauma: ${ }^{26-28}$

Abaixo, ressalta-se como se examina a inervação da mão:

- NERVO RADIAL. Motor: extensão de polegar e MFs. Sensitivo: dorso da primeira comissura;

- NERVO MEDIANO. Motor: oponência do polegar e pinça com flexão das polpas dos primeiro e segundo dedos. Sensitivo: polpa da falange distal do segundo dedo; e

- NERVO ULNAR. Motor: adução/abdução de dedos e adução do polegar. Sensitivo: borda ulnar do quinto dedo.

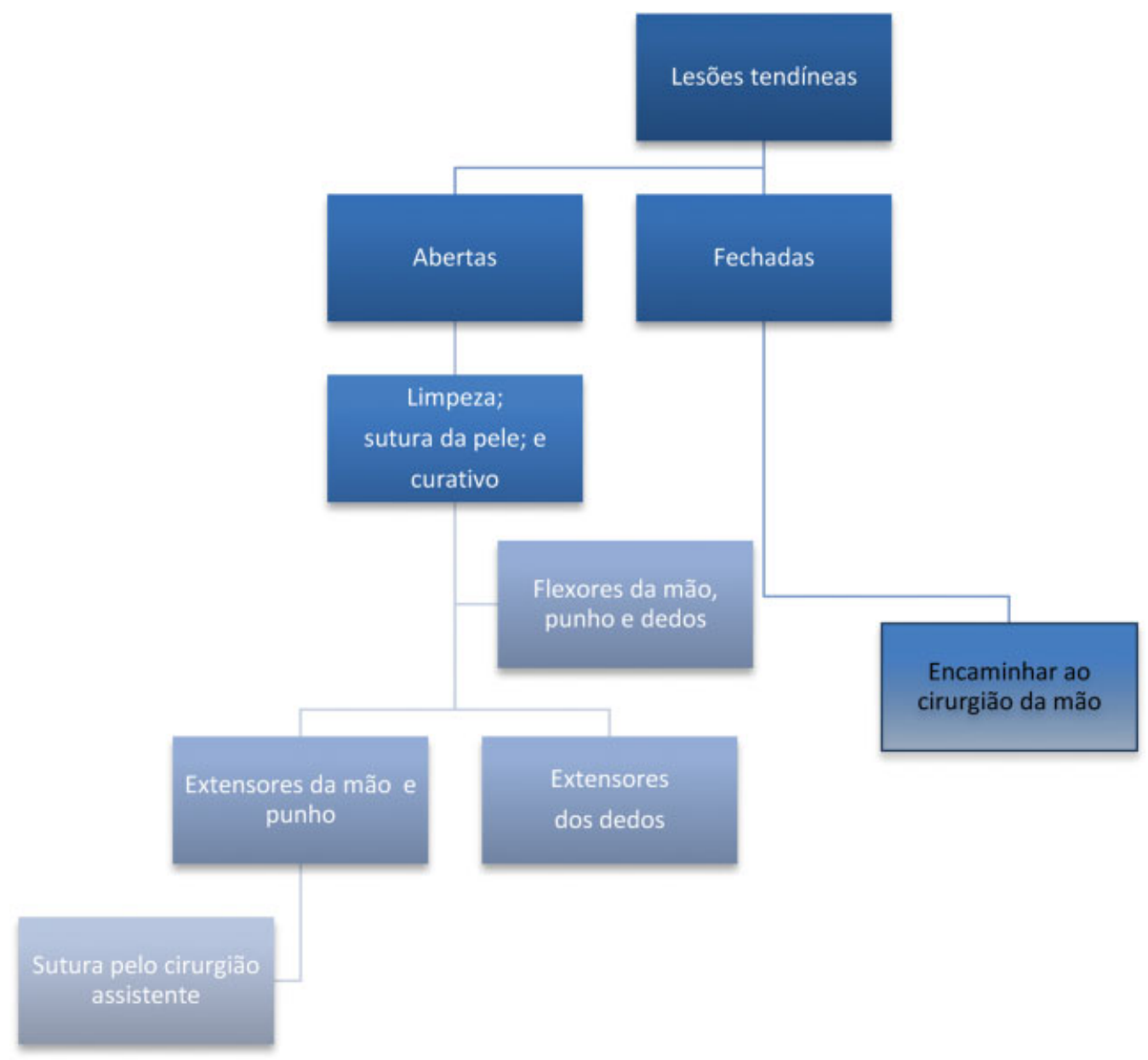

Fig. 1 Orientação de conduta para lesão tendínea. 


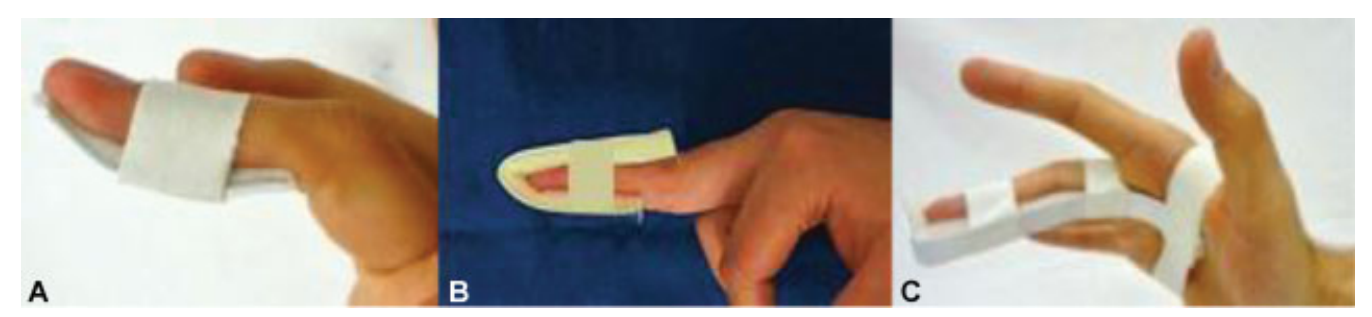

Fig. 2 (A) Tala em hiperextensão (dedo em martelo). (B) Tala em "U" para falange distal. (C) Tala em semiflexão.

\section{Fraturas}

\section{Falanges}

As fraturas de falange apresentam maior incidência entre as fraturas em geral, de $19 \%$ e $28 \%$, inclusive no esporte. ${ }^{4}$ Parte considerável dessas fraturas terá resultado satisfatório no tratamento conservador, desde que se respeitem as suas particularidades e os desvios aceitáveis para eventuais angulações. ${ }^{29}$ Quando intrarticulares, podem ter relação com subluxação nas IFs, mais bem evidenciadas na radiografia de perfil do dedo. Fraturas-avulsões da base volar, nas falanges média e distal (inserção dos flexores superficial e profundo, respectivamente), têm grande potencial de instabilidade e subluxação, especialmente se o fragmento ósseo for superior a $40 \%$ dessa articulação. ${ }^{29,30}$ Se estáveis, são mais bem tratadas com tala dorsal, bloqueando a extensão da respectiva articulação.

As fraturas extrarticulares de falanges podem sofrer a ação de inserção tendinosa da região, resultando em desvios angulares. A - Tabela 1 relaciona fraturas nas falanges com o mecanismo de trauma, a possibilidade de tratamento conservador, e a imobilização sugerida ( - Tabela $\mathbf{1}$ ).

Durante o tratamento conservador, especialmente nas duas primeiras semanas, atentar para a relação entre o local da fratura e as inserções tendíneas. O bloqueio da ação muscular evita o desvio e a perda de redução da fratura. 0 tempo de imobilização compreende três a quatro semanas, necessárias para a consolidação clínica dessas fraturas.

Alguns padrões de fratura têm indicação para redução e fixação cirúrgicas:

- Extrarticulares instáveis após redução e imobilização;

- Intrarticulares com desvio, ou instáveis após redução;
- Intrarticulares luxadas ou subluxadas (fragmento maior do que $40 \%$ da articulação em radiografias em perfil); e

- Fraturas expostas.

Quando há indicação para redução, boa parte das fraturas de falanges evolui ao bom resultado com redução fechada e fixação percutânea (com fio de Kirschner de $1,0 \mathrm{~mm}$ ) mantida por cerca de quatro semanas. Porém, podem ser utilizados placas e parafusos de tamanho específico para falanges, que são preferidos para o tratamento de lesões articulares, múltiplas e complexas, pois conferem mais estabilidade e permitem a mobilidade precoce dos dedos. O trauma de partes moles agregado e a curva de aprendizado necessária para dominar adequadamente a técnica devem ser considerados na utilização dessas sínteses. ${ }^{30-32}$

\section{Metacarpais}

O mecanismo de lesão comum na fratura do metacarpal envolve traumas axiais ou compressivos. ${ }^{33}$ Pode cursar com lesões cutânea e tendínea associadas, em geral ocasionadas por traumas cortocontundentes no dorso. O local e o desvio da fratura, sua associação com outros metacarpais, a lesão de partes moles, e a exposição óssea norteiam os limites para o tratamento conservador.

São limites para o tratamento conservador:

- AUSÊNCIA de desvios rotacionais ou sagitais;

- Encurtamento < $5 \mathrm{~mm}$;

- Primeiro, quarto e quinto metacarpos: desvios de até $30^{\circ}$ (em radiografia em perfil); e

- Segundo e terceiro metacarpos: desvios até $10^{\circ}$ (em radiografia em perfil).

Tabela 1 Relação entre a falange de dedos, o mecanismo de trauma, o padrão da fratura / desvios aceitáveis, e a imobilização sugerida no tratamento conservador de fraturas extrartriculares

\begin{tabular}{|l|l|l|l|}
\hline Falanges & $\begin{array}{l}\text { Mecanismo de trauma } \\
\text { mais comum }\end{array}$ & Padrão da fratura & Tipo de imobilização \\
\hline Distal & Axial ou compressivo & $\begin{array}{l}\text { Tofo (extremidade); } \\
\text { longitudinal; sem desvio }\end{array}$ & $\begin{array}{l}\text { Tala de alumínio em “U” } \\
\text { até a interfalangeana proximal (-Figura 2) }\end{array}$ \\
\hline $\begin{array}{l}\text { Média } \\
\text { (diáfise) }\end{array}$ & Torcional ou direto & $\begin{array}{l}\text { Rotacional: } 0^{\circ} ; \\
\text { plano coronal: }<5^{\circ} ; \\
\text { plano sagital: }<10^{\circ}\end{array}$ & $\begin{array}{l}\text { Tala de alumínio em “U” até a } \\
\text { metacarpofalangeana }\end{array}$ \\
\hline $\begin{array}{l}\text { Proximal } \\
\text { (diáfise) }\end{array}$ & Torcional & $\begin{array}{l}\text { Rotacional: } 0^{\circ} ; \\
\text { plano coronal: }<5^{\circ} ; \\
\text { plano sagital: }<10^{\circ}\end{array}$ & $\begin{array}{l}\text { Tala de alumínio volar, em semiflexão } \\
\text { da metacarpofalangeana (-Figura 2) }\end{array}$ \\
\hline
\end{tabular}


Em relação às fraturas diafisárias, a mobilidade mais ampla das articulações carpometacarpais de primeiro, quarto e quinto raios contribui para a aceitação de desvios maiores, comparada aos raios fixos, sem prejuízo na função de pinça e na força de preensão.

As fraturas no colo dos metacarpais com angulações volares acentuadas podem causar o efeito de pseudogarra por alteração no vetor de ação do tendão extensor comum dos dedos. A manobra de Jahss auxilia na redução de fraturas desviadas. $^{31}$ Os desvios volares da cabeça dos metacarpais considerados aceitáveis são diferentes para cada raio, sendo de $15^{\circ}$ para o segundo e terceiro, entre $30^{\circ}$ e $40^{\circ}$ para o quarto e, entre $50^{\circ}$ e $60^{\circ}$ para o quinto raio..$^{30}$

Fraturas intrarticulares MFs podem ser tratadas conservadoramente, desde que o desvio não seja superior a $1 \mathrm{~mm}$ e o acometimento da superfície articular seja menor do que $25 \%$.

Fraturas que acometem a base dos metacarpais têm associação frequente com instabilidade ou luxação carpometacarpal devido às inserções de tendíneas e ligamentos carpais. Raramente terão resultado satisfatório com o tratamento conservador. ${ }^{30,31}$

Quando houver indicação de tratamento cirúrgico para fratura em um dos segmentos de metacarpais, poderão ser utilizados placas, parafusos específicos, e fios de Kirschner. A escolha dependerá de fatores anteriormente abordados e da experiência do cirurgião.

Para o tratamento conservador, orienta-se a imobilização com os seguintes parâmetros: punho em extensão de $0^{\circ}$ a $30^{\circ}$; MFs em flexão de $70^{\circ}$; permitir mobilidade das IFs; e interpor gaze entre os dedos a fim de evitar lesões de pele. $^{30,31,33}$

\section{Carpo}

Fraturas de ossos carpais geralmente são causadas por queda com a mão espalmada, em que o escafoide se coloca em posição de impacto, abrangendo cerca de $60 \%$ a $70 \%$ das fraturas nessa região. ${ }^{34-36}$ Devido à estrutura e à vascularização peculiar, fraturas nesse osso podem não ser visualizadas nas radiografias seguidas ao trauma. Se a clínica for positiva (dor na palpação dorsal e palmar do escafoide e pistonagem positiva do polegar de encontro ao escafoide), mesmo com radiografias não conclusivas, o punho deve ser imobilizado por precaução até o retorno em 7 a 15 dias. ${ }^{35-37}$

Exames de tomografia e ressonância magnética são utilizados em alguns casos para identificar a fratura do escafoide, considerando-se os custos e a demanda funcional do paciente. A fratura de tratamento conservador e bom prognóstico ocorre no colo ou polo distal do osso, é estável, e não apresenta desvio. A fixação óssea está indicada para fraturas instáveis (com desvio $>1,0 \mathrm{~mm}$, fragmentadas, ou do polo proximal) nas quais há risco de pseudartrose e consequente artrose radiocarpal. ${ }^{35-37}$

Fraturas do escafoide associadas a lesões de outros ossos do carpo, luxações, ou fraturas distais do rádio são instáveis e originadas por trauma de alta energia. Essas lesões graves e complexas têm indicação cirúrgica com estabilização e reconstrução ligamentar. ${ }^{35-37}$
Fraturas isoladas em outros ossos do carpo são incomuns, e não costumam apresentar desvios. De forma geral, o tratamento é satisfatório com uso de imobilização simples por quatro semanas.

\section{Lesões ligamentares}

Entorses em IFs e MFs são traumas comuns na mão, e podem estar associadas a pequenas fraturas articulares, diagnosticadas com radiografias adequadas. Afastada a possibilidade de fraturas e após exame comparativo da estabilidade articular, a imobilização por duas semanas é suficiente para bom resultado. ${ }^{38} \mathrm{O}$ edema constante nas IFs e MFs leva à rigidez e disfunção. Portanto, nessas entorses, é preferível tratamento que permita mobilidade articular, mesmo que parcial, como a solidarização com o dedo adjacente (buddy taping), por exemplo.

Rupturas ligamentares completas são raras. Diagnosticada a instabilidade com o teste do estresse articular (que também pode ser feito durante a radiografia), haverá indicação de reparo cirúrgico, principalmente em áreas de carga, como ocorre na ruptura do ligamento colateral ulnar na MF do polegar.

Luxações em IFs e MFs necessitam de redução sob anestesia, visando relaxar estruturas que possam estar interpostas e que impedem a redução. ${ }^{38,39}$ Após congruência articular, manter tala por 15 a 20 dias, evitando o movimento que causou a luxação.

As luxações carpometacarpais e intercarpais podem passar despercebidas. Com frequência, estão relacionadas a traumas de alta energia. Deve-se atentar ao exame clínico criterioso, solicitar projeções radiográficas apropriadas, e observar angulações corretas entre metacarpais, ossos do carpo, e os terços distais do rádio e da ulna (-Figura 3). ${ }^{40}$ Radiografia comparativa do lado não acometido auxilia no diagnóstico dessas lesões graves, tratadas com reconstrução cirúrgica.

\section{Lesões múltiplas e complexas}

Lesões complexas na mão envolvem múltiplas estruturas ou até mesmo amputação de segmentos. Há relação com alta energia e risco de comprometimento da função. A avaliação emergencial deve seguir o conceito de atendimento ao politraumatizado (ATLS).

As medidas essenciais na primeira abordagem incluem:

1. Controle de sangramento

Devido ao calibre dos vasos na região da mão, com frequência a hemostasia já está contida pela formação de trombos. Pressão sobre a ferida por alguns minutos ou curativo oclusivo com o membro elevado devem ser empregados na vigência de sangramento ativo. A ligadura de vasos na mão ou punho na sala de emergência é contraindicada, pois há risco de envolver estruturas como nervos e tendões. ${ }^{41}$

2. Antibioticoterapia

Recomenda-se o uso de cefalosporina de primeira geração (para organismos Gram-positivos) e gentamicina (para 

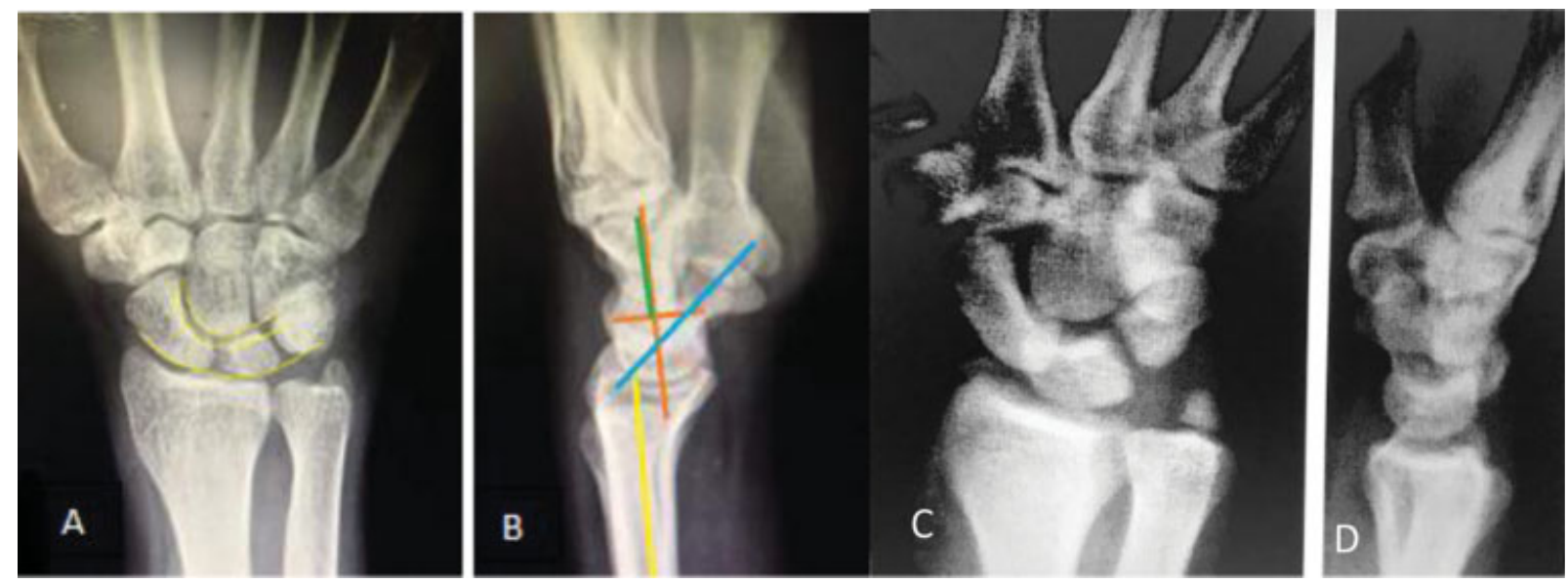

Fig. 3 (A) Arcos de Gilula e (B) eixos dos ossos carpais com o rádio (necessários para a medição de desalinhamentos e luxações). (C e D) Radiografias em projeção anteroposterior (AP) e em perfil de uma fratura-luxação perilunar, com acometimento de estiloide radial e ulnar. Notese a quebra dos arcos e a perda das angulações normais.

organismos Gram-negativos). Incluir penicilina no caso de ambientes rurais. ${ }^{42,43}$

3. Debridamento

Debridamento deve ser realizado em centro cirúrgico sob anestesia adequada, de maneira cautelosa, respeitando o limite de tecido vivo, sangrante. Boa cicatrização tecidual será atingida se esse procedimento for realizado de forma pertinente. $^{41,42,44}$

4. Estabilização óssea

A estabilização osteoarticular, seja temporária ou definitiva, é parte essencial da primeira abordagem. Permite o alinhamento ósseo, preserva a vascularização, reduz o edema, e previne a infecção. Fios de Kirschner são recomendados para esse propósito inicial. ${ }^{41,42,44}$

5. Procedimentos complementares

Após a realização do primeiro atendimento emergencial, novos procedimentos poderão ser necessários conforme demanda. Curativos sob anestesia após 24 a 48 horas são apropriados para a reavaliação e a programação das próximas etapas. Fixação definitiva das lesões osteo-articulares, reparo neurotendíneo e cobertura cutânea serão definidos nos próximos dias, de acordo com a gravidade da lesão e as condições clínicas do paciente.

\section{Considerações Finais}

Com relação aos traumatismos na mão, salienta-se alguns aspectos importantes na abordagem:

- Mobilização: orientar mobilidade precoce da mão e dedos;

- Drenagem: intercalar períodos de elevação do membro, e evitar síndrome compartimental; e

- Pele: proteger com gaze as áreas de contato (principalmente entre os dedos), orientar cuidados com curativos.

O número de pacientes com traumas na mão que procuram o pronto atendimento é expressivo, e os traumas compreendem uma gama variada de lesões. Cabe ao plantonista observar as particularidades de cada lesão, com atenção especial à anatomia, promovendo o primeiro atendimento adequado e evitando, assim, consequências irreversíveis à função da mão.

\section{Suporte Financeiro \\ Os autores declaram não haver apoio financeiro.}

Conflito de Interesses

Os autores declaram não haver conflito de interesses.

\section{Referências}

1 de Putter CE, Selles RW, Polinder S, Panneman MJ, Hovius SE, van Beeck EF. Economic impact of hand and wrist injuries: health-care costs and productivity costs in a population-based study. J Bone Joint Surg Am 2012;94(09):e56

2 Junqueira GDR, Lima ALM, Boni R, Almeida JC, Ribeiro RS, Figueiredo LA. Incidence of acute trauma on hand and wrist: a retrospective study. Acta Ortop Bras 2017;25(06):287-290

3 Ribak S, de Oliveira EJN, Rosolino GP, Orru P, Tietzmann A. Epidemiology of traumatic injuries of the upper limbs in a university hospital. Acta Ortop Bras 2018;26(06):370-373

4 Hile D, Hile L. The emergent evaluation and treatment of hand injuries. Emerg Med Clin North Am 2015;33(02):397-408

5 Singh R, Chojnowski A, Hay S. Hand and Wrist Injuries Related to Motocross Injuries: 5 Year Series. J Hand Surg Asian Pac Vol 2019; 24(01):60-64

6 Luria S, Talmud D, Volk I, Liebergall M, Calderon-Margalit R. The epidemiology of wrist and hand injury in two hospitals in Jerusalem: substantial differences between population subgroups. Isr J Health Policy Res 2019;8(01):7-18

7 Schneuer FJ, Bell JC, Adams SE, Brown J, Finch C, Nassar N. The burden of hospitalized sports-related injuries in children: an Australian population-based study, 2005-2013. Inj Epidemiol 2018;5(01):45-55

8 Lemme NJ, Ready L, Faria M, DeFroda SF, Gil JA, Owens BD. Epidemiology of boxing-related upper extremity injuries in the United States. Phys Sportsmed 2018;46(04):503-508

9 Eisele A, Dereskewitz C, Kus S, Oberhauser C, Rudolf KD, Coenen MConsortium Lighthouse Project Hand. Factors affecting time off work in patients with traumatic hand injuries-A bio-psychosocial perspective. Injury 2018;49(10):1822-1829

10 Gyer G, Michael J, Inklebarger J. Occupational hand injuries: a current review of the prevalence and proposed prevention 
strategies for physical therapists and similar healthcare professionals. J Integr Med 2018;16(02):84-89

11 Rosberg HE, Dahlin LB. An increasing number of hand injuries in an elderly population - a retrospective study over a 30 -year period. BMC Geriatr 2018;18(01):68-75

12 Siotos C, Ibrahim Z, Bai J, et al. Hand injuries in low- and middleincome countries: systematic review of existing literature and call for greater attention. Public Health 2018;162:135-146

13 Wong KY, Maw J, Gillespie P. Assessment of hand injuries. Br J Hosp Med (Lond) 2016;77(03):C41-C44

14 Ciclamini D, Panero B, Titolo P, Tos P, Battiston B. Particularities of hand and wrist complex injuries in polytrauma management. Injury 2014;45(02):448-451

15 Kitay A, Wolfe SW. Scapholunate instability: current concepts in diagnosis and management. J Hand Surg Am 2012;37(10): 2175-2196

16 Gyftopoulos S, Chitkara M, Bencardino JT. Misses and errors in upper extremity trauma radiographs. AJR Am J Roentgenol 2014; 203(03):477-491

17 Pederson WC. Nonmicrosurgical Coverage of the Upper Extremity. In: Wolfe SW, Hotchkiss RN, Pederson WC, Kozin SH, Cohen MS, eds. Green's Operative Hand Surgery. Philadelphia: Elsevier; 2017:1528-1573

18 Nellans KW, Chung KC. Pediatric hand fractures. Hand Clin 2013; 29(04):569-578

19 Markiewitz AD. Complications of hand fractures and their prevention. Hand Clin 2013;29(04):601-620

20 Sommer NZ. Perionychium. In: Wolfe SW, Hotchkiss RN, Pederson WC, Kozin SH, Cohen MS, editors. Green's Operative Hand Surgery. 7th ed. Philadelphia: Elsevier; 2017:318-337

21 Loréa P. Primary care of nail traumas. Chir Main 2013;32(03): 129-135

22 Silva JB, Gerhardt S. Trauma to the nail complex. Rev Bras Ortop 2014;49(02):111-115

23 George A, Alexander R, Manju C. Management of Nail Bed Injuries Associated with Fingertip Injuries. Indian J Orthop 2017;51(06): 709-713

24 Lin JS, Samora JB. Surgical and Nonsurgical Management of Mallet Finger: A Systematic Review. J Hand Surg Am 2018;43(02): 146-163.e2

25 Çapkın S, Buyuk AF, Sürücü S, Bakan OM, Atlihan D. Extensionblock pinning to treat bony mallet finger: Is a transfixation pin necessary? Ulus Travma Acil Cerrahi Derg 2019;25(03):281-286

26 Yildiran G, Sutcu M, Akdag O, Tosun Z. Long-Term Outcomes of Digital Nerve Repair Accompanied by Digital Artery Injury in Flexor Zone 2. Surg J (N Y) 2019;6(01):e7-e9

27 Jain A, Dunlop R, Hems T, Tang JB. Outcomes of surgical repair of a single digital nerve in adults. J Hand Surg Eur Vol 2019;44(06): 560-565

28 Dunlop RLE, Wormald JCR, Jain A. Outcome of surgical repair of adult digital nerve injury: a systematic review. BMJ Open 2019;9 (03):e025443
29 Oetgen ME, Dodds SD. Non-operative treatment of common finger injuries. Curr Rev Musculoskelet Med 2008;1(02): 97-102

30 Henry MH. Hand Fractures and Dislocations. In: Court-Brown CM, Heckman JD, McQueen MM, Ricci WM, Tornetta P, McKee MD, eds. Rockwood and Green's Fractures in Adults. 8th ed. Philadelphia2015:915-990

31 Day CS. Fractures of Metacarpals and Phalanges. In: Wolfe SW, Hotchkiss RN, Pederson WC, Kozin SH, Cohen MS, eds. Green's Operative Hand Surgery. 7th ed. Philadelphia: Elsevier; 2017: 231-277

32 Richards T, Clement R, Russell I, Newington D. Acute hand injury splinting - the good, the bad and the ugly. Ann R Coll Surg Engl 2018;100(02):92-96

33 Ramirez EG, Hoyt KS. Management of Hand Injuries: Part II. Adv Emerg Nurs J 2016;38(04):266-278

34 Duckworth AD, Jenkins PJ, Aitken SA, Clement ND, Court-Brown CM, McQueen MM. Scaphoid fracture epidemiology. J Trauma Acute Care Surg 2012;72(02):E41-E45

35 Fowler JR, Hughes TB. Scaphoid fractures. Clin Sports Med 2015; 34(01):37-50

36 Andrew D. Duckworth, Ring D. Carpus Fractures and Dislocations. In: Court-Brown CM, Heckman JD, McQueen MM, Ricci WM, Tornetta P, McKee MD, eds. Rockwood and Green's Fractures in Adults. 8th ed. Philadelphia: Elsevier; 2015:991-1056

37 Steve K. Lee. Fracture of the Carpal Bones. In: Wolfe SW, Hotchkiss RN, Pederson WC, Kozin SH, Cohen MS, eds. Green's Operative Hand Surgery. 7th ed. Philadelphia: Elsevier; 2017:588-652

38 Rozmaryn LM. The Collateral Ligament of the Digits of the Hand: Anatomy, Physiology, Biomechanics, Injury, and Treatment. J Hand Surg Am 2017;42(11):904-915

39 Merrell G, Hastings H. Dislocations and Ligament Injuries of the Digits. In: Wolfe SW, Hotchkiss RN, Pederson WC, Kozin SH, Cohen MS, eds. Green's Operative Hand Surgery. 7th ed. Philadelphia: Elsevier; 2017:278-317

40 Garcia-Elias M, Lluch AL. Wrist Instabilities, Misalignments, and Dislocations. In: Wolfe SW, Hotchkiss RN, Pederson WC, Kozin SH, Cohen MS, eds. Green's Operative Hand Surgery. 7th ed. Philadelphia: Elsevier; 2017:418-478

41 Tosti R, Eberlin KR. “Damage Control” Hand Surgery: Evaluation and Emergency Management of the Mangled Hand. Hand Clin 2018;34(01):17-26

42 Friedrich JB, Vedder NB. Mangled Upper Extremity. In: Wolfe SW, Hotchkiss RN, Pederson WC, Kozin SH, Cohen MS, eds. Green's Operative Hand Surgery. 7th ed. Philadelphia: Elsevier; 2017: 1486-1527

43 Warrender WJ, Lucasti CJ, Chapman TR, Ilyas AM. Antibiotic Management and Operative Debridement in Open Fractures of the Hand and Upper Extremity: A Systematic Review. Hand Clin 2018;34(01):9-16

44 Agarwal R, Agarwal D, Agarwal M. Approach to mutilating hand injuries. J Clin Orthop Trauma 2019;10(05):849-852 